\title{
Fulvestrant and the sequential endocrine cascade for advanced breast cancer
}

\author{
S Johnston*,I \\ 'Department of Medicine (Breast Unit), The Royal Marsden Hospital NHS Trust \& Institute of Cancer Research, Fulham Road, London SW3 6J), UK
}

\begin{abstract}
Following relapse on endocrine therapy for advanced, hormone receptor-positive breast cancer, it is common for patients to experience responses to alternative endocrine agents. Fulvestrant ('Faslodex') is a new type of endocrine treatment - an oestrogen receptor (ER) antagonist with no agonist effects. Fulvestrant downregulates cellular levels of the ER resulting in decreased expression of the progesterone receptor. This unique mode of action means that it is important that fulvestrant is placed optimally within the sequence of endocrine therapies to ensure that patients gain maximum benefit. Fulvestrant has shown efficacy when used after progression on tamoxifen or anastrozole in postmenopausal women with advanced breast cancer. After progression on fulvestrant, subsequent endocrine treatments can produce responses in many patients, demonstrating that fulvestrant does not lead to crossresistance with other endocrine therapies. Responses to fulvestrant have also been observed in patients heavily pretreated with prior endocrine therapy. Fulvestrant is a versatile endocrine agent that may be integrated into the therapeutic sequence prior to, or subsequent to, other hormonal therapies, and represents a valuable additional antioestrogen for the treatment of postmenopausal women with advanced breast cancer.

British Journal of Cancer (2004) 90(SuppI I), SI5-SI8. doi:I0.1038/sj.bjc.660I632 www.bjcancer.com

(C) 2004 Cancer Research UK
\end{abstract}

Keywords: breast cancer; endocrine therapy; sequencing; fulvestrant; 'Faslodex'

The efficacy and tolerability advantages associated with the use of endocrine agents in the treatment of hormone receptor-positive advanced breast cancer have been clearly established in many clinical studies. However, despite an initial response, many patients will eventually experience disease progression and require further endocrine treatment options. In patients who respond to endocrine treatments, additional responses to further agents are common (Buzdar and Hortobagyi, 1998; Hortobagyi, 1998). This potential responsiveness to multiple endocrine therapies means that patients may continue to derive clinical benefit while avoiding the marked, and often distressing, adverse side effects associated with chemotherapy. This is a particularly important consideration in a predominantly elderly patient population who may be least able to tolerate severe adverse events. Disease control is also important in this patient group for whom an absolute cure may not be achievable, and instead, prevention of disease progression and the maintenance of quality of life may be more important.

The activity of sequential endocrine therapies is dependent upon them possessing different mechanisms of action. In this way, crossresistance between sequential therapies may be avoided. It is therefore important that, as new endocrine therapies with different mechanisms of action become available, they are integrated effectively into the sequential hormonal regimens to allow patients to derive maximum benefit.

\footnotetext{
*Correspondence: Dr SRD Johnston;

E-mail: Stephen.johnston@rmh.nthames.nhs.uk
}

\section{FULVESTRANT AND THE ENDOCRINE SEQUENCE CASCADE}

Fulvestrant ('Faslodex') is a new type of endocrine treatment - an oestrogen receptor (ER) antagonist with no agonist effects (Wakeling et al, 1991; Robertson et al, 2001). Fulvestrant binds to the ER but, due to its steroidal structure and long side-chain, induces a different conformational shape with the receptor to that achieved by the nonsteroidal antioestrogen tamoxifen. As a result of this, fulvestrant prevents ER dimerisation and leads to the rapid degradation of the fulvestrant-ER complex, producing the loss of cellular ER (Borras et al, 1996). As a result, fulvestrant (unlike tamoxifen) inhibits ER - DNA binding and produces abrogation of oestrogen-sensitive gene transcription (Dauvois et al, 1993).

The unique mode of action of fulvestrant presents a useful addition to the endocrine agents currently available for use in sequential therapeutic regimens. Fulvestrant has been approved in the United States and Brazil for the treatment of hormone receptor-positive metastatic breast cancer in postmenopausal women with disease progression following antioestrogen therapy. An increasing number of studies are demonstrating the versatility of fulvestrant for the treatment of advanced breast cancer (Howell et al, 2002; Osborne et al, 2002; Perey et al, 2002; Steger et al, 2003a, b). An understanding and appreciation of these data will be important for determining the optimal placing of fulvestrant in the sequence cascade of hormonal therapy.

\section{Efficacy post-tamoxifen}

The efficacy of fulvestrant has been proven in two phase III trials conducted in postmenopausal patients with hormone-sensitive 
advanced breast cancer progressing on prior tamoxifen. In both these trials, the efficacy of fulvestrant was comparable to the highly selective, third-generation aromatase inhibitor (AI) anastrozole ('Arimidex') (Howell et al, 2002; Osborne et al, 2002). Fulvestrant is the only antioestrogen acting directly on ER that has demonstrated efficacy post-tamoxifen, illustrating the lack of crossresistance between these two therapies. This is in contrast to the selective oestrogen receptor modulators (SERMs) such as droloxifene, idoxifene, toremifene, and benzothiophene arzoxifene, all of which have shown minimal activity in tamoxifenresistant disease (Johnston, 2001).

\section{Efficacy post-AI}

Owing to their improved efficacy and tolerability, AIs are increasingly being used in the first-line treatment of breast cancer, in both early and advanced disease (Nabholtz et al, 2000; ATAC Trialists' Group, 2002; Mouridsen et al, 2003). Preclinical data indicate that exposure to long-term oestrogen deprivation (similar to that caused by AIs) and subsequent development of acquired resistance may be accompanied by adaptive increases in ER gene expression and intercellular signalling, resulting in hypersensitivity to low oestradiol levels (Jeng et al, 1998; Shim et al, 2000; Chan et al, 2002; Martin et al, 2003). In this situation, tamoxifen may be perceived as an agonist. As a result, it is important to establish the efficacy of fulvestrant after progression on AIs. In vitro, fulvestrant significantly inhibited the expression of genes such as c-myb and c-myc in cells resistant to long-term oestrogen deprivation (Jeng et al, 1998) and may therefore be an appropriate therapeutic option after progression on AIs.

Clinical data so far are limited, but preliminary results from an ongoing phase II study have shown that fulvestrant produced clinical benefit $(\mathrm{CB}$, complete response $(\mathrm{CR})+$ partial response $(\mathrm{PR})+$ stable disease $(\mathrm{SD}) \geqslant 24$ weeks) in seven out of $17(41 \%)$ patients who had received, and had progressed on, prior treatment with tamoxifen and an AI (Perey et al, 2002). These results suggest that in addition to producing responses after prior tamoxifen, disease progression after anastrozole may not preclude subsequent treatment with fulvestrant. Further trials in this setting are now in progress and are discussed later in this paper.

\section{Efficacy in heavily pretreated patients}

Many patients may receive a number of different endocrine therapies as well as chemotherapies during the course of their disease. Preliminary data are becoming available from centres using fulvestrant in Named Patient Programmes involving patients heavily pretreated with endocrine therapies including tamoxifen, anastrozole, letrozole, exemestane, and goserelin. In 67 postmenopausal women with metastatic breast cancer, 64 of whom $(96 \%)$ had progressed on one, two or three prior endocrine agents for advanced disease, fulvestrant produced CB in 40 patients $(60 \%)$ overall. A total of six patients $(9 \%)$ derived a PR. Of these, one had received fulvestrant as first-line therapy for advanced disease, two had received fulvestrant as second-line therapy, and three had received it as third-line therapy. No objective responses were seen in patients receiving fourth-line fulvestrant therapy. This might suggest that fulvestrant produces better responses when given earlier in the treatment sequence (Steger et al, 2003a).

Similar results have been obtained in a separate single-centre study. Postmenopausal women with metastatic breast cancer who had been heavily pretreated with prior hormonal therapy (including tamoxifen, AIs, androgens, and high-dose oestrogens) and chemotherapy (including taxanes, capecitabine, doxorubicin, and cisplatin) were treated with fulvestrant; SD $\geqslant 24$ weeks was achieved in eight out of 42 (19\%) patients (Franco et al, 2003).

\section{Endocrine therapy after progression on fulvestrant}

Two studies have provided evidence that the marked reduction in ER expression produced by fulvestrant is not associated with crossresistance to subsequent endocrine therapies (Howell, 2002; Vergote et al, 2003). These studies used the retrospective analysis of data derived from questionnaires sent to clinicians who were involved in trials of fulvestrant as first- or second-line therapy (Osborne et al, 2002; Robertson et al, 2002). This methodology imposes certain limitations on the analyses. However, the information obtained from these studies does provide further evidence with regard to establishing sequencing regimens.

Responses to subsequent endocrine therapy in patients who progressed on fulvestrant or tamoxifen as first-line therapy for advanced disease have been examined in a retrospective analysis (Howell, 2002). The limitations of this analysis are illustrated by the fact that while 170 patients derived CB on fulvestrant, followup data on patients who received subsequent endocrine therapy were available for only 35 of these. In these patients, subsequent endocrine therapy produced CB in 20 out of $35(57 \%)$ patients, with AI-based therapy producing CB in 11 out of $22(50 \%)$ patients (Table 1). It is interesting to note that in those patients who failed to derive $\mathrm{CB}$ from fulvestrant, subsequent endocrine therapy produced a similar number of responses to those seen in patients who did derive CB from fulvestrant (15 out of $35(43 \%)$ and 20 out of $35(57 \%)$, respectively; Table 2$)$. While the selection of patients and patient numbers included in this analysis are limited by the nature of the data collection, they do indicate that responses may be obtained with AIs and other endocrine therapies after progression on fulvestrant.

In another retrospective analysis, 186 patients in total derived $\mathrm{CB}$ on second-line fulvestrant, although questionnaire-based follow-up data were available for only 54 of these patients (Vergote et al, 2003). The results showed that treatment with endocrine therapy (predominantly AIs) after second-line fulvestrant produced CB in 25 out of $54(46 \%)$ patients and objective response $(\mathrm{OR}, \mathrm{CR}+\mathrm{PR})$ in four out of $54(7 \%)$ patients who obtained $\mathrm{CB}$ with second-line fulvestrant (Table 1). In patients who failed to derive $\mathrm{CB}$ from second-line fulvestrant, further endocrine therapy produced CB in 18 out of $51(35 \%)$ patients and OR in one out of $51(2 \%)$ patients (Table 2). Preliminary analyses demonstrated a median duration of response to subsequent therapy of 383 and 318 days, for patients who did and who did not derive CB from secondline fulvestrant, respectively. Further endocrine therapy after progression on fulvestrant is therefore a viable and effective therapeutic option, with responses seen in patients treated with

Table I Response to subsequent therapy in patients who derived clinical benefit (CB) from fulvestrant

\begin{tabular}{|c|c|c|c|c|c|}
\hline & \multicolumn{5}{|c|}{ Number of patients } \\
\hline & $\mathbf{C R}$ & PR & $S D \geqslant 24$ weeks & PD & Total \\
\hline \multicolumn{6}{|c|}{ Patients who derived CB from first-line fulvestrant } \\
\hline Endocrine therapy total & I & 2 & 17 & 15 & 35 \\
\hline Aromatase inhibitors & I & । & 9 & 11 & 22 \\
\hline Tamoxifen & 0 & I & 7 & 2 & 10 \\
\hline Megestrol acetate & 0 & 0 & i & 0 & । \\
\hline Medroxyprogesterone acetate & 0 & 0 & 0 & 2 & 2 \\
\hline \multicolumn{6}{|c|}{ Patients who derived CB from second-line fulvestrant } \\
\hline Endocrine therapy total & 0 & 4 & 21 & 29 & 54 \\
\hline Aromatase inhibitors & 0 & 3 & 16 & 27 & 46 \\
\hline Megestrol acetate & 0 & । & 5 & 2 & 8 \\
\hline
\end{tabular}


tamoxifen and megestrol acetate as well as AIs such as anastrozole and letrozole.

\section{DISCUSSION}

It is important to be aware of the sequence versatility of fulvestrant so that it may be effectively and appropriately incorporated into the endocrine sequence cascade. Fulvestrant has demonstrated efficacy in the treatment of postmenopausal women with advanced, hormone-sensitive breast cancer, with data indicating that fulvestrant exhibits this activity in both the post-tamoxifen

Table 2 Response to subsequent therapy in patients who did not derive clinical benefit (CB) from fulvestrant

\begin{tabular}{lccccc}
\hline & \multicolumn{5}{c}{ Number of patients } \\
\cline { 2 - 6 } & CR & PR & SD $\geqslant \mathbf{2 4}$ weeks & PD & Total \\
\hline Patients who did not derive CB from first-line fulvestrant \\
Endocrine therapy total & 0 & 3 & 12 & 20 & 35 \\
Aromatase inhibitors & 0 & 0 & 8 & 11 & 19 \\
Tamoxifen & 0 & 3 & 2 & 7 & 12 \\
Megestrol acetate & 0 & 0 & $\mid$ & 0 & 1 \\
Medroxyprogesterone acetate & 0 & 0 & 1 & 2 & 3 \\
Patients who did not derive CB from second-line fulvestrant & \\
Endocrine therapy total & 0 & $\mid$ & 17 & 33 & 51 \\
Aromatase inhibitors & 0 & $\mid$ & 15 & 26 & 42 \\
Megestrol acetate & 0 & 0 & $\mid$ & 5 & 6 \\
Medroxyprogesterone acetate & 0 & 0 & $\mid$ & 2 & 3 \\
\hline
\end{tabular}

Adapted from Howell (2002) with permission of Breast Cancer Research and Treatment. (Vergote et al, 2003). $C R=$ complete response; $P R=$ partial response; $\mathrm{SD}=$ stable disease; $\mathrm{PD}=$ progressive disease.



$\mathrm{Al}=$ aromatase inhibitor

$\mathrm{MA}=$ megestrol acetate

Figure I Proposed positions of fulvestrant within the available endocrine therapies for the sequential treatment of postmenopausal women with hormone receptor-positive, advanced disease. Adapted from Carlson (2002) with permission of Breast Cancer Research and Treatment. and postanastrozole setting. More specifically, fulvestrant has been shown to be at least as effective as anastrozole in women with hormone-sensitive disease who have progressed on first-line therapy (mainly tamoxifen) (Howell et al, 2002; Osborne et al, 2002; Robertson et al, 2003), with preliminary data showing promising results after progression on AIs (Perey et al, 2002). Fulvestrant has also been used in patients pretreated with several endocrine agents as well as chemotherapy. In one report, an overall $\mathrm{CB}$ rate of $60 \%$ was obtained, although patients who were treated with fulvestrant earlier in the sequence appeared to obtain better responses than those who received it after progression on three endocrine agents (Steger et al, 2003a).

In patients who undergo disease progression on fulvestrant, the novel mode of action of this new endocrine therapy ensures a lack of crossresistance to other current endocrine agents (Howell, 2002; Vergote et al, 2003). Thus, the early use of fulvestrant in the sequence of endocrine therapies may not limit later choices of endocrine therapy. Fulvestrant can, therefore, potentially be integrated into sequential endocrine regimens at a number of positions, including the second-line setting after tamoxifen, or, potentially, after AIs (Figure 1). Endocrine therapies such as megestrol acetate or the steroidal AI exemestane may then be employed as necessary after progression on fulvestrant. In this way, the potentially most effective and well-tolerated agents are used earlier in the treatment sequence.

To optimise the positioning of fulvestrant in the sequence of endocrine therapies, additional studies will be required to elaborate upon the data so far accrued. New phase II and III clinical trials of fulvestrant in over 3000 patients are either planned or currently in progress (Table 3 ). These will investigate additional roles for fulvestrant in breast cancer therapy, either following prior nonsteroidal AI treatment or in combination with AIs as first-line therapy. In addition, loading-dose fulvestrant regimens will be tested. Two randomised, controlled trials are comparing the efficacy and tolerability of fulvestrant $v s$ exemestane in postmenopausal women progressing after long-term oestrogen deprivation resulting from prior AI therapy. The primary aim of the Study Of Faslodex $v s$ Exemestane with/without Arimidex (SOFEA) trial is to compare progression-free survival in patients who have progressed on a nonsteroidal AI, and who are subsequently treated with either fulvestrant plus continued anastrozole, or with fulvestrant alone. Secondary aims include a comparison of fulvestrant $v s$ exemestane and an examination of biological markers of response. A further trial, the Evaluation of Faslodex vs Exemestane Clinical Trial (EFECT) is currently recruiting patients to assess the efficacy of fulvestrant $v s$ exemestane in patients who have progressed on treatment with nonsteroidal AIs. In addition, two trials (FACT and SWOG 226) will compare the efficacy of a combination of fulvestrant plus anastrozole with anastrozole alone in the first-line setting (Table 3 ). The results of

Table 3 New phase II/III clinical trials of fulvestrant in over 3000 breast cancer patients

\begin{tabular}{|c|c|c|c|c|}
\hline Trial & Phase & Population & Treatments & Patients ( $n)$ \\
\hline NCCTG & $\|$ & Post-tamoxifen or post-Als & Fulvestrant $250 \mathrm{mg}$ & 89 \\
\hline SAKK & $\|$ & Post-tamoxifen or post-Als & Fulvestrant $250 \mathrm{mg}$ & 93 \\
\hline SOFEA & III & Post-nonsteroidal Al & Fulvestrant LD $250 \mathrm{mg} \pm$ anastrozole vs exemestane & 750 \\
\hline FACT & III & First-line & Fulvestrant LD $250 \mathrm{mg}+$ anastrozole vs anastrozole & 558 \\
\hline SWOG 226 & III & First-line & Fulvestrant $250 \mathrm{mg}+$ anastrozole vs anastrozole & 690 \\
\hline 0057 & $\|$ & Neoadjuvant & Fulvestrant $250 \mathrm{mg}+$ anastrozole vs anastrozole & 120 \\
\hline
\end{tabular}

NCCTG = North Central Cancer Treatment Group; SAKK = Swiss Group for Clinical Cancer Research; EFECT=Evaluation of Faslodex vs Exemestane Clinical Trial; SOFEA = Study Of Faslodex vs Exemestane with/without Arimidex; SWOG = Southwest Oncology Group; LD $250 \mathrm{mg}=$ loading-dose schedule of fulvestrant: $500 \mathrm{mg}$ day 0 , $250 \mathrm{mg}$ days 14 and 28 , fulvestrant $250 \mathrm{mg}$ per monthly thereafter; $\mathrm{HD}=$ high-dose schedule of fulvestrant $750 \mathrm{mg} 2-3$ weeks presurgery. 
trials such as these will further define endocrine-sequencing strategies, particularly as AIs move forward into the first-line or adjuvant settings.

Currently available data therefore indicate that fulvestrant will be a useful therapeutic option that may extend the opportunity for using endocrine therapies before reliance upon cytotoxic chemotherapy is necessary. Fulvestrant is also a versatile endocrine therapy that may be used at a variety of positions in the sequential use of endocrine therapy for postmenopausal women with advanced, hormone-sensitive breast cancer.

\section{REFERENCES}

ATAC Trialists' Group (2002) Anastrozole alone or in combination with tamoxifen versus tamoxifen alone for adjuvant treatment of postmenopausal women with early breast cancer: first results of the ATAC randomised trial. Lancet 359: $2131-2139$

Borras M, Laios I, el Khissiin A, Seo HS, Lempereur F, Legros N, Leclercq G (1996) Estrogenic and antiestrogenic regulation of the half-life of covalently labeled estrogen receptor in MCF-7 breast cancer cells. Steroid Biochem Mol Biol 57: $203-213$

Buzdar AU, Hortobagyi G (1998) Update on endocrine therapy for breast cancer. Clin Cancer Res 4: $527-534$

Carlson RW (2002) Sequencing of endocrine therapies in breast cancer integration of recent data. Breast Cancer Res Treat 75(Suppl 1): S27-S32

Chan CM, Martin LA, Johnston SR, Ali S, Dowsett M (2002) Molecular changes associated with the acquisition of oestrogen hypersensitivity in MCF-7 breast cancer cells on long-term oestrogen deprivation. J Steroid Biochem Mol Biol 81: 333-341

Dauvois S, White R, Parker MG (1993) The antiestrogen ICI 182780 disrupts estrogen receptor nucleocytoplasmic shuttling. J Cell Sci 106(Part 4): $1377-1388$

Franco S, Perez A, Tan-Chiu E, Frankel C, Vogel C (2003) Fulvestrant (Faslodex ${ }^{\circledR}$ ) demonstrates clinical benefit in heavily pretreated postmenopausal women with advanced breast cancer: a single-center experience. Breast Cancer Res Treat 82(Suppl 1): S105 (abstract 429)

Hortobagyi GN (1998) Progress in endocrine therapy for breast carcinoma. Cancer 83: $1-6$

Howell A (2002) Postmenopausal women with advanced breast cancer who progress on fulvestrant or tamoxifen retain sensitivity to further endocrine therapies. Breast Cancer Res Treat 76(Suppl 1): S72 (abstract 251)

Howell A, Robertson JFR, Quaresma Albano J, Aschermannova A, Mauriac L, Kleeberg UR, Vergote I, Erikstein B, Webster A, Morris C (2002) Fulvestrant, formerly ICI 182,780, is as effective as anastrozole in postmenopausal women with advanced breast cancer progressing after prior endocrine treatment. J Clin Oncol 20: 3396-3403

Jeng MH, Shupnik MA, Bender TP, Westin EH, Bandyopadhyay D, Kumar R, Masamura S, Santen RJ (1998) Estrogen receptor expression and function in long-term estrogen-deprived human breast cancer cells. Endocrinology 139: $4164-4174$

Johnston SR (2001) Endocrine manipulation in advanced breast cancer: recent advances with SERM therapies. Clin Cancer Res 7: $4376 \mathrm{~s}-4387 \mathrm{~s}$

Martin LA, Farmer I, Johnston SR, Ali S, Marshall C, Dowsett M (2003) Enhanced estrogen receptor (ER) alpha, ERBB2, and MAPK signal transduction pathways operate during the adaptation of MCF-7 cells to long term estrogen deprivation. J Biol Chem 278: $30458-30468$

Mouridsen H, Gershanovich M, Sun Y, Perez-Carrion R, Boni C, Monnier A, Apffelstaedt J, Smith R, Sleeboom HP, Jaenicke F, Pluzanska A, Dank M, Becquart D, Bapsy PP, Salminen E, Snyder R, Chaudri-Ross H, Lang R, Wyld P, Bhatnagar A (2003) Phase III study of letrozole versus tamoxifen as first-line therapy of advanced breast cancer in postmeno- pausal women: analysis of survival and update of efficacy from the International Letrozole Breast Cancer Group. J Clin Oncol 21: 2101 - 2109

Nabholtz JM, Buzdar A, Pollak M, Harwin W, Burton G, Mangalik A, Steinberg M, Webster A, von Euler M (2000) Anastrozole is superior to tamoxifen as first-line therapy for advanced breast cancer in postmenopausal women: results of a North American multicenter randomized trial. Arimidex Study Group. J Clin Oncol 18: 3758-3767

Osborne CK, Pippen J, Jones SE, Parker LM, Ellis M, Come S, Gertler SZ, May JT, Burton G, Dimery I, Webster A, Morris C, Elledge R, Buzdar A (2002) Double-blind, randomized trial comparing the efficacy and tolerability of fulvestrant versus anastrozole in postmenopausal women with advanced breast cancer progressing on prior endocrine therapy: results of a North American trial. J Clin Oncol 20: 3386-3395

Perey L, Thürlimann B, Hawle $\mathrm{H}$, Bonnefoi $\mathrm{H}$, Ahern J, Pagani O, Goldhirsch A, Dietrich D (2002) Fulvestrant ('faslodex') as hormonal treatment in postmenopausal patients with advanced breast cancer progressing after treatment with tamoxifen and aromatase inhibitors. Breast Cancer Res Treat 76(Suppl 1): S72 (abstract 249)

Robertson JF, Nicholson RI, Bundred NJ, Anderson E, Rayter Z, Dowsett M, Fox JN, Gee JM, Webster A, Wakeling AE, Morris C, Dixon M (2001) Comparison of the short-term biological effects of 7-alpha-[9-(4,4,5,5,5pentafluoropentylsulfinyl)-nonyl] estra-1,3,5, (10)-triene-3,17beta-diol (Faslodex) versus tamoxifen in postmenopausal women with primary breast cancer. Cancer Res 61: 6739-6746

Robertson JF, Osborne CK, Howell A, Jones SE, Mauriac L, Ellis M, Kleeberg UR, Come SE, Vergote I, Gertler S, Buzdar A, Webster A, Morris C (2003) Fulvestrant versus anastrozole for the treatment of advanced breast carcinoma in postmenopausal women - a prospective combined analysis of two multicenter trials. Cancer 98: 229-238

Robertson JFR, Howell A, Abram P, Lichinitser M, Elledge R (2002) Fulvestrant versus tamoxifen for the first-line treatment of advanced breast cancer (ABC) in postmenopausal women. Ann Oncol 13(Suppl 5): 46 (abstract 1640)

Shim WS, Conaway M, Masamura S, Yue W, Wang JP, Kmar R, Santen RJ (2000) Estradiol hypersensitivity and mitogen-activated protein kinase expression in long-term estrogen deprived human breast cancer cells in vivo. Endocrinology 141: $396-405$

Steger G, Bartsch R, Wenzel C, Pluschnig U, Locker G, Mader RM, Zielinski CC (2003a) Fulvestrant ('Faslodex') demonstrates clinical benefit in heavily pre-treated patients with metastatic breast cancer. Eur J Cancer 1(Suppl 5): S135

Steger G, Bartsch R, Wenzel C, Pluschnig U, Locker G, Mader RM, Zielinski CC (2003b) Fulvestrant beyond the second hormonal treatment line in metastatic breast cancer. Proc Am Soc Clin Oncol 22: 20

Vergote I, Robertson JFR, Kleeberg U, Burton G, Osborne CK, Mauriac L (2003) Postmenopausal women who progress on fulvestrant ('Faslodex') remain sensitive to further endocrine therapy. Breast Cancer Res Treat 79: $207-211$

Wakeling AE, Dukes M, Bowler J (1991) A potent specific pure antiestrogen with clinical potential. Cancer Res 51: $3867-3873$ 